\title{
An assessment of a semi analytical AG method for solving nonlinear oscillators
}

\author{
Hadi Mirgolbabaee, Soheil Tahernejad Ledari and Davood Domiri Ganji \\ Department of Mechanical Engineering, Babol University of Technology, Babol, Iran
}

Received: 17 June 2015, Revised: 24 June 2015, Accepted: 17 August 2015

Published online: 21 February 2016.

\begin{abstract}
In this paper, attempts have been made to solve nonlinear vibrational equation such as Van Der Pol Oscillator by utilizing a semi analytical Akbari-Ganji's Method (AGM). It is noticeable that there are some valuable advantages in this way of solving differential equations and also the answer of various sets of complicated differential equations can be achieved in this manner which in the other methods it would be difficult to obtain.

Based on the comparison between AGM and numerical methods, AGM can be successfully applied for a broad range of nonlinear equations. One of the important reasons of selecting AGM for solving differential equations in miscellaneous fields not only in vibrations but also in different fields of sciences for instance fluid mechanics, solid mechanics, chemical engineering, etc. The main benefit of this method in comparison with the other approaches are as follows, normally according to the order of differential equations, we need boundary conditions so in the case of the number of boundary conditions is less than the order of the differential equation, AGM can create additional new boundary conditions in regard to the own differential equation and its derivatives. Results illustrate that method is efficient and has enough accuracy in comparison with other semi analytical and numerical methods because of the simplicity of this method. Moreover results demonstrate that AGM could be applicable through other methods in nonlinear problems with high nonlinearity. Furthermore convergence problems for solving nonlinear equations by using AGM appear small.
\end{abstract}

Keywords: Akbari-Ganji method (AGM), nonlinear equations, nonlinear oscillator, Van Der Pol oscillator.

\section{Introduction}

In this case study, attempts have been made to analyze the vibration equations of nonlinear oscillators by a powerful analytical method which is named Akbari-Ganji's Method (AGM) [1,2,3]. The main purpose of this investigation is introducing AGM as an efficient method for solving nonlinear equation in vibrational equation by solving the problems (which is mentioned in other sections) with different assumption of trial functions as the answer of the equations.

Most scientific problems such as vibrational problems are inherently nonlinear and currently studies through this equations has been considered more because there are many practical engineering components consisting of oscillating systems which can be modeled by using oscillatory systems . Except a limited number of these problems, most of them don't have analytical solution. Therefore, these nonlinear equations should be solved using other methods. In addition to all the benefits of using numerical methods, closed form solutions appear more appealing because they reveal physical insights through the physics of the problem.

An overview of the published papers reveals that different authors have utilized different analytical methods for solving nonlinear equations especially in the field of vibrations in order to analyze oscillation systems. Some of these well-known methods can be mentioned as, Homotopy Analysis Method [4,5], the He's Amplitude Frequency 
Formulation method (HAFF) [6,7], Parameter-Expansion Mthod [8], Energy Balance Method [9, 10], Differential Transformation Method (DTM)[11], Homotopy Perturbation Method [12,13], Adomian Decomposition Method [14,15], EXP-function Method [16,17,18,19] and Variational Iteration Method by J.H.He [20,21]. But the afore-mentioned methods do not have this ability to gain the solution of the presented problem in high precision. Therefore, these nonlinear equations should be solved by using other approaches.

It is necessary to mention that a summary of the excellence of this method in comparison with the other approaches can be considered as follows, Boundary conditions are needed in accordance with the order of differential equations in the solution procedure but when the number of boundary conditions is less than the order of the differential equation, this approach can create additional new boundary conditions in regard to the own differential equation and its derivatives. Therefore, it is logical to mention that AGM is operational for miscellaneous nonlinear differential equations in comparison with the other methods.

\section{Basic idea of Akbari-Ganji's method}

In general, vibrational equations and their initial conditions are defined for different systems as follows

$$
f\left(\ddot{u}, \dot{u}, u, F_{0} \sin \left(\omega_{0} t\right)\right)=0 .
$$

Parameter $\left(\omega_{0}\right)$ angular frequency of the harmonic force exerted on the system and $\left(F_{0}\right)$ the maximum amplitude its.And initial conditions as follows

$$
\left\{u(t)=u_{0}, \quad \dot{u}(t)=0, \quad \text { at } \quad t=0\right\} .
$$

\subsection{Choosing the answer of the governing equation for solving differential equations by AGM}

In AGM, a total answer with constant coefficients is required in order to solve differential equations in various fields of study such as vibrations, structures, fluids and heat transfer. In vibrational systems with respect to the kind of vibration, it is necessary to choose the mentioned answer in AGM. To clarify here, we divide vibrational systems into two general forms.

\section{Vibrational systems without any external force}

Differential equations governing on this kind of vibrational systems are introduced in the following form

$$
f(\ddot{u}, \dot{u}, u)=0 \text {. }
$$

Now, the answer of this kind of vibrational system is chosen as

$$
u(t)=e^{-a t}\{A \cos (\omega t)+B \sin (\omega t)\} .
$$

According to trigonometric relationships, Eq.(4) is rewritten as follows:

$$
u(t)=e^{-a t}\{b \cos (\omega t+\varphi)\} .
$$

(c) 2016 BISKA Bilisim Technology 
It is notable that in the Eq. (5), $b=\sqrt{A^{2}+B^{2}}, \varphi=\arctan \left(\frac{B}{A}\right)$.

Sometimes for increasing the precision of the considered answer of Eq. (3), we are able to add another term in the form of cosine by inspiration of Fourier cosine series expansion as follows:

$$
u(t)=e^{-a t}\left\{b \cos \left(\omega t+\varphi_{1}\right)+d \cos \left(2 \omega t+\varphi_{2}\right)\right\} .
$$

In the above equation, we are able to omit the term $\left(e^{-a t}\right)$ to facilitate the computational operations in AGM if the system is considered without any damping components.

Generally speaking in AGM, Eq. (5) or Eq. (6) is assumed as the answer of the vibrational differential equation (3) that its constant coefficients which are $a, b, d, \omega$ (angular frequency) and $\varphi$ (initial vibrational phase) can easily be obtained by applying the given initial conditions in Eq. (2). And also the above procedure will completely be explained through the presented example in the foregoing part of the paper. It is noteworthy that if there is no damping component in the vibrational system, the constant coefficient $a$ in Eq. (5) and Eq. (6) will automatically be computed zero in AGM solution procedure.

On the contrary, the parameter bin Eq. (5) and Eq. (6) for the other kind of vibrational system with damping component is obtained as a nonzero parameter in AGM.

\section{Vibrational systems with external force}

In this step, it is assumed that the external forces exerting on the vibrational systems are defined as

$$
F(t)=F_{0} \sin \left(\omega_{0} t\right)
$$

As a result, the differential equation governing on the vibrational system is expressed like Eq. (1) as follows

$$
f\left(\ddot{u}, \dot{u}, u, F_{0} \sin \left(\omega_{0} t\right)\right)=0 .
$$

The answer of the above equation is introduced as the sum of the particular solution $\left(u_{p}\right)$ and the harmonic solution $\left(u_{h}\right)$ as follows

$$
\begin{gathered}
u_{h}(t)=e^{-a t}\{A \cos (\omega t)+B \sin (\omega t)\} \\
u_{p}(t)=M \cos \left(\omega_{0} t\right)+N \sin \left(\omega_{0} t\right) .
\end{gathered}
$$

The result answer differential equation Eq.(8) as follows

$$
u(t)=u_{p}+u_{h}
$$

By utilizing trigonometric relationships $\left\{b=\sqrt{A^{2}+B^{2}}, d=\sqrt{M^{2}+N^{2}}\right\}$ and $\left\{\varphi=\arctan \left(\frac{B}{A}\right), \phi=\arctan \left(\frac{N}{M}\right)\right\}$ also by substituting the yielded equations into Eq. (10), the desired answer will be obtained in the form of

$$
u(t)=e^{-a t}\{b \cos (\omega t+\varphi)\}+d \cos \left(\omega_{0} t+\phi\right)
$$


In order to increase the precision of the achieved equation, we are able to add another term in the form of cosine by inspiration of Fourier cosine series expansion as follows

$$
u(t)=e^{-a t}\left\{b \cdot \cos \left(\omega t+\varphi_{1}\right)+c \cdot \cos \left(2 \omega t+\varphi_{2}\right)\right\}+d \cdot \cos \left(\omega_{0} t+\phi\right) .
$$

And finally in accordance with the Eq. (12), the exact solution of the all vibrational differential equations can be obtained in the following equation

$$
u(t)=e^{-a t}\left\{\sum_{k=1}^{\infty} b_{k} \cos \left(k \omega t+\varphi_{k}\right)\right\}+d \cos \left(\omega_{0} t+\phi\right) .
$$

The constant coefficients of Eq.(13) which are $\left\{a_{1}, a_{2}, \ldots, \varphi_{1}, \varphi_{2}, \ldots, b, \omega, d, \phi\right\}$ will easily be computed in AGM by applying the initial conditions of Eq.(2). To deeply understand the above procedure, reading the following lines is recommended.

Since the constant coefficient $(b)$ in vibrational systems without damping components is always obtained zero $(a=0)$ which in the case, to decrease computational operations of Eq.(12) and (or Eq.(13)) in the following form:

$$
\left\{\begin{array}{l}
u(t)=\left\{b \cdot \cos \left(\omega t+\varphi_{1}\right)+c \cdot \cos \left(2 \omega t+\varphi_{2}\right)\right\}+d \cdot \cos \left(\omega_{0} t+\phi\right) \\
o r \quad u(t)=\left\{\sum_{k=1}^{\infty} b_{k} \cos \left(k \omega t+\varphi_{k}\right)\right\}+d \cos \left(\omega_{0} t+\phi\right)
\end{array}\right\} .
$$

Based on the above explanations, by applying initial conditions on a system without damping component, the value of parameter $(a)$ is always zero for Eqs. (11-13). Therefore without damping component, the role of parameter $(a)$ in the both of Eq. (11-13) which each of them can be considered as the answer of the vibrational problems is individually considered as a catalyst for increasing the precision of the assumed answer. However according to Eqs.(11-13) after applying initial conditions on the vibrational system in both states (with external force and without external force) by AGM, the value of parameter $(a)$ is computed zero because the mentioned system has a free vibration without any damping component.

Again, we mention that in order to decrease computational operations for systems without damping components and since we know that $(a)$ in the term $\left(e^{-a t}\right)$ is zero so $\left(e^{-a t}\right)$ can be omitted from Eqs. (11-13). Consequently, Eq. (11) which has been considered as the answer of the systems without any damping component can be rewritten as follows

$$
u(t)=b \cdot \cos (\omega t+\varphi)+d \cdot \cos \left(\omega_{0} t+\phi\right) .
$$

\section{Application of initial conditions to compute constant coefficients and angular frequency by AGM}

In AGM, the application of initial conditions of Eq.(2) is done in the two following forms

(a) Applying the initial conditions on the answer of differential equation. In regard to the kind of vibrational system (with external force and without external force) which was completely discussed in the previous part of this case study, a function is chosen as the answer of the differential equation from Eq.(5) or Eq.(6) for the systems without external forces and from Eqs.(11-15) for the defined systems with external forces and then the initial conditions are applied on the selected function as follows:

$$
u(t)=u(I C) .
$$

It is notable that IC is the abbreviation of introduced initial conditions of Eq.(2). 
(b) Appling the initial conditions on the main differential equation and its derivatives. After choosing a function as the answer of differential equation according to the kind of vibrational system, this is the best time to substitute the mentioned answer into the main differential equation instead of its dependent variable $(u)$.

Assume the general equation of the vibration such as Eq.(1) with time-independent parameter $(t)$ and dependent function $(u)$ as

$$
f\left(\ddot{u}, \dot{u}, u, F_{0} \sin \left(\omega_{0} t\right)\right)=0 .
$$

Therefore on the basis of the kind of vibrational system, a function as the answer of the differential equation such as Eq.(5) or Eq.(6) and Eqs.(11-15) are considered as follows

$$
u=g(t) .
$$

In this step, the afore-mentioned equation is substituted into Eq.(17) instead of $(u)$ in the following form

$$
f(t)=f\left(g^{\prime}(t), g^{\prime}(t), g(t), F_{0} \sin \left(\omega_{0} t\right)\right) .
$$

Eventually, the application of initial conditions on Eq.(19) and its derivatives is expressed as

$$
\begin{aligned}
& f(I C)=f\left(g^{\prime \prime}(I C), g^{\prime}(I C), g(I C), \ldots\right), \\
& f^{\prime}(I C)=f^{\prime}\left(g "(I C), g^{\prime}(I C), g(I C), \ldots\right), \\
& f^{\prime \prime}(I C)=f^{\prime \prime}\left(g "(I C), g^{\prime}(I C), g(I C), \ldots\right), \ldots \ldots
\end{aligned}
$$

To end up, it is better to say that in AGM after applying the initial conditions on answer function Eq.(16), and also the function differential equation and on its derivatives from Eq.(20) according to the order of differential equation and utilizing the two given initial conditions of Eq.(2), a set of algebraic equations which is consisted of $n$ equations with $n$ unknowns is created. Therefore, the constant coefficients $(a, b, c, d$, angular frequency $(\omega)$ and initial phase $(\varphi)$ and $\phi)$ at Eqs. $(11,12)$ are easily achieved which this procedure will thoroughly be explained in the form of an example in the foregoing part of this paper.

It is noteworthy that in Eq.(19), we are able to use the derivatives of $f(t)$ with higher orders until the number of yielded equations is equal to the number of the mentioned constant coefficients of the assumed answer.

\section{Application}

Example 1. In accordance with the following picture, consider the Van Der Pol Oscillator problem [22,23] with its nonlinear equation as below

$$
\frac{d^{2}}{d t^{2}} u(t)+\frac{d}{d t} u(t)+u(t)+u^{2}(t)\left(\frac{d}{d t} u(t)\right)=2 \cos (t)-\cos ^{3}(t)
$$

The initial conditions are expressed in the forms of

$$
u(0)=0, u^{\prime}(0)=1 .
$$




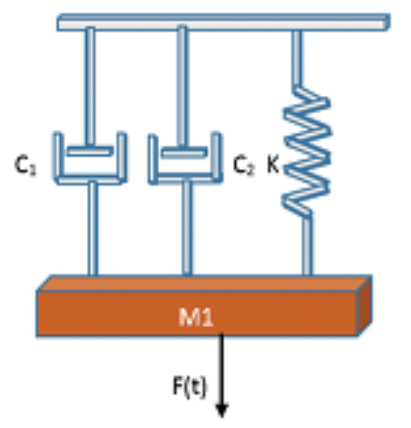

Fig. 1: Geometry of example 1.

\subsection{Solving the differential equation with AGM}

For solving the above equation, first of all we rewrite the problem Eq.(21) in the following order

$$
g(t): \frac{d^{2}}{d t^{2}} u(t)+\frac{d}{d t} u(t)+u(t)+u^{2}(t) \cdot\left(\frac{d}{d t} u(t)\right)=2 \cdot \cos (t)-\cos ^{3}(t) .
$$

On the basis of the given explanations in the previous section, the answer of Eq.(22) in AGM is considered as follows:

$$
u(t)=e^{-a t}(b \cos (\omega t+\varphi))
$$

In AGM, the constant coefficients of Eq.(24) which are $\omega$ (angular frequency), $a, b$ and $\varphi$ (initial vibrational phase) can easily be computed by applying initial conditions or boundary conditions according to the physical aspects of the problem.

\subsection{Applying boundary or initial conditions}

In accordance with the proposed physical model, there are no boundary conditions so the constant coefficients of Eq. (24) are achieved just with respect to the given initial conditions which are presented in Eq. (22). It is notable that initial or boundary conditions are applied in two manners in the following forms

(a) The initial conditions are applied on Eq.(24) in the form of

$$
u=u(I C)
$$

To simplify, IC is considered as the abbreviation of the initial conditions.

As a result, applying the initial conditions on Eq.(24) is done as

$$
\begin{gathered}
\mathrm{u}(0)=0 \rightarrow \mathrm{b} \cos (\varphi)=0, \\
u^{\prime}(0)=1 \rightarrow-a \cdot b \cos (\varphi)-b \sin (\varphi) \omega=1 .
\end{gathered}
$$

(b) The application of initial or boundary conditions on the main differential equation which in this case is Eq.(23) and its derivatives is done in the following general forms

$$
g(u(t)) \rightarrow g(u(I C))=0, g^{\prime}(u(I C))=0, \ldots
$$


Therefore according to Eq.(28), the initial conditions are applied after substituting Eq.(24) which was considered as the answer of the main differential equation into Eq.(23).

As a result, the above procedure is done on the yielded equation and its derivative as

$$
\begin{aligned}
& g(u(0)): a^{2} b \cos (\varphi)+2 a b \sin (\varphi) \omega-b \cos (\varphi) \omega^{2}-a b \cos (\varphi) \\
& -b \sin (\varphi) \omega+b \cos (\varphi)+b^{2} \cos ^{2}(\varphi)(-a b \cos (\varphi)-b \sin (\varphi) \omega)=1 .
\end{aligned}
$$

And then, we have

$$
\begin{aligned}
& g^{\prime}(u(0)):-a^{3} b \cos (\varphi)-3 a^{2} b \sin (\varphi) \omega+3 a b \cos (\varphi) \omega^{2}+b \sin (\varphi) \omega^{3} \\
& +a^{2} b \cos (\varphi)+2 a b \sin (\varphi) \omega-b \cos (\varphi) \omega^{2}-a b \cos (\varphi)-b \sin (\varphi) \omega \\
& -2 b^{2} \cos ^{2}(\varphi)(-a b \cos (\varphi)-b \sin (\varphi) \omega) a-2 b^{2} \cos (\varphi)(-a b \cos (\varphi)-b \sin (\varphi) \omega) \\
& \sin (\varphi) \omega+b^{2} \cos ^{2}(\varphi)\left(a^{2} b \cos (\varphi)+2 a b \sin (\varphi) \omega-b \cos (\varphi) \omega^{2}\right)=0 .
\end{aligned}
$$

By solving a set of algebraic equations which is consisted of four equations with four unknowns from Eqs.(26-27) and Eqs.(29-30), the constant coefficients $a, b, \varphi$, and $\omega$ of Eq. (24) can easily be yielded as follows:

$$
a=0, b=1, \omega=1, \varphi=-1.570796327 \text {. }
$$

After substituting the obtained values from Eq.(31) and into Eq.(24), the solution of the mentioned problem will be obtained as follows

$$
u(t)=\cos (t-1.570796327)
$$

Example 2. with consideration of the following figure consider the nonlinear equation [24] as below

$$
\frac{d^{2}}{d t^{2}} x(t)+\alpha \cdot x(t)+\varepsilon \cdot x^{3}(t)=0
$$

The initial conditions are introduced in the forms of

$$
x(0)=A, x^{\prime}(0)=0 .
$$

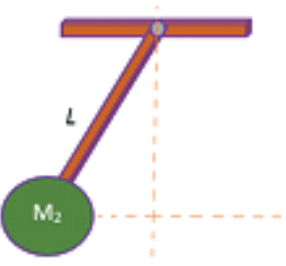

Fig. 2: Geometry of example 2. 


\subsection{Solving the differential equation with AGM}

For solving the above nonlinear equation first all we rewrite the problem Eq.(33) in the following order

$$
f(t): \frac{d^{2}}{d t^{2}} x(t)+\alpha x(t)+\varepsilon x^{3}(t)=0 .
$$

To solve the nonlinear differential equation by AGM, it is necessary to consider a function as the answer of the problem which is explained the previous part, so we consider the answer of the problem as follows:

$$
u(t)=e^{-a t}\left(b_{0} \cos \left(\omega t+\varphi_{0}\right)+b_{1} \cos \left(2 \omega t+\varphi_{1}\right)+b_{2} \cos \left(3 \omega t+\varphi_{2}\right)\right) .
$$

On the basis of the given explanations, the existence of the term $e^{-a \cdot t}$ in Eq. (36) indicates that there is a damping component in the oscillating system. Since there are not any damping components in the mentioned example, the term $a$ in Eq.(36) will automatically be zero after applying the initial conditions in AGM. Moreover, the constant coefficient $b$, the initial vibrational phase $\varphi$ and finally the angular frequency $\omega$ can be computed by applying the initial conditions. According to above theory the answer of the problem is assumed as following

$$
u(t)=b_{0} \cos \left(\omega t+\varphi_{0}\right)+b_{1} \cos \left(2 \omega t+\varphi_{1}\right)+b_{2} \cos \left(3 \omega t+\varphi_{2}\right)
$$

\subsection{Applying boundary or initial conditions}

In regard to the proposed physical model, there are no boundary conditions so the constant coefficients of Eq.(37) are just acquired with respect to the given initial conditions which have been presented in Eq.(34). It is notable that initial or boundary conditions are applied in two manners in the following form

(a) The initial conditions are applied on Eq.(37) in the form of

$$
x=x(I C) .
$$

To simplify, IC is considered as the abbreviation of the initial conditions.

As a result, applying the initial conditions on Eq.(37) is done as

$$
x(0)=A \rightarrow b_{0} \cos \left(\varphi_{0}\right)+b_{1} \cos \left(\varphi_{1}\right)+b_{2} \cos \left(\varphi_{2}\right)=A .
$$

And

$$
x^{\prime}(0)=0 \rightarrow-b_{0} \sin \left(\varphi_{0}\right) \omega-2 b_{1} \sin \left(\varphi_{1}\right) \omega-3 b_{2} \sin \left(\varphi_{2}\right) \omega=0 .
$$

(b) The application of initial or boundary conditions on the main differential equation which in this case is Eq. (35) and its derivatives is done in the following general forms

$$
f\left(x^{\prime}(t)\right) \rightarrow f\left(x^{\prime}(I C)\right)=0, f^{\prime}\left(x^{\prime}(I C)\right)=0, \ldots
$$

Therefore according to Eq.(41), the initial conditions are applied after substituting Eq.(37) which was considered as the answer of the main differential equation into Eq.(35). 
As a result, the above procedure is done on the yielded equation and its derivative as:

$$
\begin{aligned}
& f\left(x^{\prime}(0)\right):-b_{0} \cos \left(\varphi_{0}\right) \omega^{2}-4 b_{1} \cos \left(\varphi_{1}\right) \omega^{2}-9 b_{2} \cos \left(\varphi_{2}\right) \omega^{2}+\alpha\left(\left(b_{0} \cos \left(\varphi_{0}\right)+b_{1} \cos \left(\varphi_{1}\right)+b_{2} \cos \left(\varphi_{2}\right)\right)\right) \\
& +\varepsilon\left(\left(b_{0} \cos \left(\varphi_{0}\right)+b_{1} \cos \left(\varphi_{1}\right)+b_{2} \cos \left(\varphi_{2}\right)\right)\right)^{3}=0, \\
& f^{\prime}\left(x^{\prime}(0)\right): b_{0} \sin \left(\varphi_{0}\right) \omega^{3}+8 b_{1} \sin \left(\varphi_{1}\right) \omega^{3}+27 b_{2} \sin \left(\varphi_{2}\right) \omega^{3}+\alpha\left(\left(-b_{0} \sin \left(\varphi_{0}\right) \omega-2 b_{1} \sin \left(\varphi_{1}\right) \omega-3 b_{2} \sin \left(\varphi_{2}\right) \omega\right)\right) \\
& +3 \varepsilon\left(\left(b_{0} \cos \left(\varphi_{0}\right)+b_{1} \cos \left(\varphi_{1}\right)+b_{2} \cos \left(\varphi_{2}\right)\right)\right)^{2}\left(\left(\left(\left(-b_{0} \sin \left(\varphi_{0}\right) \omega-2 b_{1} \sin \left(\varphi_{1}\right) \omega-3 b_{2} \sin \left(\varphi_{2}\right) \omega\right)\right)\right)\right)=0, \\
& f^{\prime \prime}\left(x^{\prime}(0)\right): b_{0} \cos \left(\varphi_{0}\right) \omega^{4}+16 b_{1} \cos \left(\varphi_{1}\right) \omega^{4}+81 b_{2} \cos \left(\varphi_{2}\right) \omega^{4}+\alpha\left(\left(-b_{0} \cos \left(\varphi_{0}\right) \omega^{2}-4 b_{1} \cos \left(\varphi_{1}\right) \omega^{2}\right.\right. \\
& \left.\left.-9 b_{2} \cos \left(\varphi_{2}\right) \omega^{2}\right)\right)+6 \varepsilon\left(\left(b_{0} \cos \left(\varphi_{0}\right)+b_{1} \cos \left(\varphi_{1}\right)+b_{2} \cos \left(\varphi_{2}\right)\right)\right)\left(\left(-b_{0} \sin \left(\varphi_{0}\right) \omega-2 b_{1} \sin \left(\varphi_{1}\right) \omega\right.\right. \\
& \left.\left.-3 b_{2} \sin \left(\varphi_{2}\right) \omega\right)\right)^{2}+3 \varepsilon\left(\left(b_{0} \cos \left(\varphi_{0}\right)+b_{1} \cos \left(\varphi_{1}\right)+b_{2} \cos \left(\varphi_{2}\right)\right)\right)^{2}\left(\left(-b_{0} \cos \left(\varphi_{0}\right) \omega^{2}-4 b_{1} \cos \left(\varphi_{1}\right) \omega^{2}\right.\right. \\
& \left.\left.-9 b_{2} \cos \left(\varphi_{2}\right) \omega^{2}\right)\right)=0 \text {, } \\
& f^{\prime \prime \prime}\left(x^{\prime}(0)\right):-b_{0} \sin \left(\varphi_{0}\right) \omega^{5}-32 b_{1} \sin \left(\varphi_{1}\right) \omega^{5}-243 b_{2} \sin \left(\varphi_{2}\right) \omega^{5}+\alpha\left(\left(b_{0} \sin \left(\varphi_{0}\right) \omega^{3}+8 b_{1} \sin \left(\varphi_{1}\right) \omega^{3}\right.\right. \\
& \left.\left.+27 b_{2} \sin \left(\varphi_{2}\right) \omega^{3}\right)\right)+6 \varepsilon\left(\left(-b_{0} \sin \left(\varphi_{0}\right) \omega-2 b_{1} \sin \left(\varphi_{1}\right) \omega-3 b_{2} \sin \left(\varphi_{2}\right) \omega\right)\right)^{3} \\
& +18 \varepsilon\left(\left(b_{0} \cos \left(\varphi_{0}\right)+b_{1} \cos \left(\varphi_{1}\right)+b_{2} \cos \left(\varphi_{2}\right)\right)\right)\left(\left(-b_{0} \sin \left(\varphi_{0}\right) \omega-2 b_{1} \sin \left(\varphi_{1}\right) \omega\right.\right. \\
& \left.\left.-3 b_{2} \sin \left(\varphi_{2}\right) \omega\right)\right)\left(\left(-b_{0} \cos \left(\varphi_{0}\right) \omega^{2}-4 b_{1} \cos \left(\varphi_{1}\right) \omega^{2}-9 b_{2} \cos \left(\varphi_{2}\right) \omega^{2}\right)\right) \\
& +3 \varepsilon\left(\left(b_{0} \cos \left(\varphi_{0}\right)+b_{1} \cos \left(\varphi_{1}\right)+b_{2} \cos \left(\varphi_{2}\right)\right)\right)^{2}\left(\left(b_{0} \sin \left(\varphi_{0}\right) \omega^{3}+8 b_{1} \sin \left(\varphi_{1}\right) \omega^{3}\right.\right. \\
& \left.\left.+27 b_{2} \sin \left(\varphi_{2}\right) \omega^{3}\right)\right)=0 \text {, } \\
& f^{(4)}\left(x^{\prime}(0)\right):-b_{0} \cos \left(\varphi_{0}\right) \omega^{6}-64 b_{1} \cos \left(\varphi_{1}\right) \omega^{6}-729 b_{2} \cos \left(\varphi_{2}\right) \omega^{6}+\alpha\left(\left(b_{0} \cos \left(\varphi_{0}\right) \omega^{4}+16 b_{1} \cos \left(\varphi_{1}\right) \omega^{4}\right.\right. \\
& \left.\left.+81 b_{2} \cos \left(\varphi_{2}\right) \omega^{4}\right)\right)+36 \varepsilon\left(\left(-b_{0} \sin \left(\varphi_{0}\right) \omega-2 b_{1} \sin \left(\varphi_{1}\right) \omega-3 b_{2} \sin \left(\varphi_{2}\right) \omega\right)\right)^{2}\left(\left(-b_{0} \cos \left(\varphi_{0}\right) \omega^{2}\right.\right. \\
& \left.\left.-4 b_{1} \cos \left(\varphi_{1}\right) \omega^{2}+-9 b_{2} \cos \left(\varphi_{2}\right) \omega^{2}\right)\right)+18 \varepsilon\left(\left(b_{0} \cos \left(\varphi_{0}\right)+b_{1} \cos \left(\varphi_{1}\right)+b_{2} \cos \left(\varphi_{2}\right)\right)\right) \\
& \left(\left(-b_{0} \cos \left(\varphi_{0}\right) \omega^{2}-4 b_{1} \cos \left(\varphi_{1}\right) \omega^{2}-9 b_{2} \cos \left(\varphi_{2}\right) \omega^{2}\right)\right)^{2}+24 \varepsilon\left(\left(b_{0} \cos \left(\varphi_{0}\right)+b_{1} \cos \left(\varphi_{1}\right)+b_{2} \cos \left(\varphi_{2}\right)\right)\right) \\
& \left(\left(-b_{0} \sin \left(\varphi_{0}\right) \omega-2 b_{1} \sin \left(\varphi_{1}\right) \omega-3 b_{2} \sin \left(\varphi_{2}\right) \omega\right)\right)\left(\left(b_{0} \sin \left(\varphi_{0}\right) \omega^{3}+8 b_{1} \sin \left(\varphi_{1}\right) \omega^{3}+27 b_{2} \sin \left(\varphi_{2}\right) \omega^{3}\right)\right) \\
& +3 \varepsilon\left(\left(b_{0} \cos \left(\varphi_{0}\right)+b_{1} \cos \left(\varphi_{1}\right)+b_{2} \cos \left(\varphi_{2}\right)\right)\right)^{2}\left(\left(b_{0} \cos \left(\varphi_{0}\right) \omega^{4}+16 b_{1} \cos \left(\varphi_{1}\right) \omega^{4}\right.\right. \\
& \left.\left.+81 b_{2} \cos \left(\varphi_{2}\right) \omega^{4}\right)\right)=0 \text {. }
\end{aligned}
$$

By solving a set of algebraic equations which is consisted of seven equations with seven unknowns from Eqs.(39, 40) and Eqs.(42, 46), the constant coefficients $b_{0}, b_{1}, b_{2}, \varphi_{0}, \varphi_{1}, \varphi_{2}$ and $\omega$ of Eq.(37) can easily be yielded as follows. To simplify, the following new variables are introduced as

$$
\begin{aligned}
& \psi=256645 A^{6} \varepsilon^{3}+467319 A^{4} \alpha \varepsilon^{2}+234687 A^{2} \alpha^{2} \varepsilon+24013 \alpha^{3} \\
& +324 \sqrt{\begin{array}{l}
720150 A^{12} \varepsilon^{6}+2447310 A^{10} \alpha \varepsilon^{5}+3206796 A^{8} \alpha^{2} \varepsilon^{4}+2090172 A^{6} \alpha^{3} \varepsilon^{3} \\
+747246 A^{4} \alpha^{4} \varepsilon^{2}+135510 A^{2} \alpha^{5} \varepsilon-1200 \alpha^{6}
\end{array}} \\
& \Gamma=\frac{1}{108} \psi^{\frac{1}{3}}-\frac{108\left(\frac{2135}{11664} A^{4} \varepsilon^{2}+\frac{623}{5832} A^{2} \alpha \varepsilon-\frac{889}{11664} \alpha^{2}\right)}{\psi^{\frac{1}{3}}}+\frac{49}{108} A^{2} \varepsilon+\frac{49}{108} \alpha .
\end{aligned}
$$




$$
\begin{aligned}
& \kappa_{1}=-\frac{4093119}{2} A^{14} \alpha \varepsilon^{7}-\frac{34744149}{2} A^{12} \alpha^{2} \varepsilon^{6}-\frac{49237215}{2} A^{10} \alpha^{3} \varepsilon^{5}-\frac{27101207}{2} A^{8} \alpha^{4} \varepsilon^{4} \\
& -\frac{4808853}{2} A^{6} \alpha^{5} \varepsilon^{3}+\frac{828547}{6} A^{4} \alpha^{6} \varepsilon^{2}+\frac{473}{6} A^{2} \alpha^{7}+36949526 A^{8} \Gamma \alpha^{3} \varepsilon^{4}-15507288 A^{8} \Gamma^{2} \alpha^{2} \varepsilon^{4} \\
& +68335560 A^{10} \Gamma \alpha^{2} \varepsilon^{5}-19462032 A^{10} \Gamma^{2} \alpha \varepsilon^{5}+53917182 A^{12} \Gamma \alpha \varepsilon^{6}-\frac{2488}{3} A^{2} \Gamma \alpha^{6} \varepsilon \\
& -7661304 A^{12} \Gamma^{2} \varepsilon^{6}+2256 A^{2} \Gamma^{2} \alpha^{5} \varepsilon+15724800 A^{14} \Gamma \varepsilon^{7}-\frac{26}{3} \Gamma \alpha^{7}+24 \Gamma^{2} \alpha^{6}-\frac{2069474}{3} A^{4} \Gamma \alpha^{5} \varepsilon^{2} \\
& +\frac{3}{2} \alpha^{8}+\frac{3448575}{2} A^{16} \varepsilon^{8}+605880 A^{4} \Gamma^{2} \alpha^{4} \varepsilon^{2}+6126560 A^{6} \Gamma \alpha^{4} \varepsilon^{3}-3109824 A^{6} \Gamma^{2} \alpha^{3} \varepsilon^{3} . \\
& \zeta_{1}=\frac{\sqrt{\kappa_{1}} A}{\left(27 A^{4} \varepsilon^{2}+24 A^{2} \alpha \varepsilon+\alpha^{2}\right)^{2}} \\
& \begin{aligned}
\kappa_{2}= & -16936992 A^{14} \alpha \varepsilon^{7}-24564672 A^{12} \alpha^{2} \varepsilon^{6}-14036448 A^{10} \alpha^{3} \varepsilon^{5}+53648 A^{8} \alpha^{4} \varepsilon^{4} \\
& +3124128 A^{6} \alpha^{5} \varepsilon^{3}+\frac{26977568}{3} A^{4} \alpha^{6} \varepsilon^{2}-\frac{16096}{3} A^{2} \alpha^{7} \varepsilon-20516512 A^{8} \Gamma \alpha^{3} \varepsilon^{4} \\
& +14275440 A^{8} \Gamma^{2} \alpha^{2} \varepsilon^{4}-8157024 A^{10} \Gamma \alpha^{2} \varepsilon^{5}+7991136 A^{10} \Gamma^{2} \alpha \varepsilon^{5}+4567392 A^{12} \Gamma \alpha \varepsilon^{6} \\
& +\frac{132640}{3} A^{2} \Gamma \alpha^{6} \varepsilon+1442448 A^{12} \Gamma^{2} \varepsilon^{6}-38688 A^{2} \Gamma^{2} \alpha^{5} \varepsilon+3241728 A^{14} \Gamma \varepsilon^{7} \\
& +\frac{800}{3} \Gamma \alpha^{7}-240 \Gamma^{2} \alpha^{6}-\frac{9696352}{3} A^{4} \Gamma \alpha^{5} \varepsilon^{2}-\frac{80}{2} \alpha^{8}-4240512 A^{16} \varepsilon^{8}+2358000 A^{4} \Gamma^{2} \alpha^{4} \varepsilon^{2} \\
& -14300672 A^{6} \Gamma \alpha^{4} \varepsilon^{3}+10095552 A^{6} \Gamma^{2} \alpha^{3} \varepsilon^{3}
\end{aligned}
\end{aligned}
$$$$
\zeta_{2}=\frac{\sqrt{\kappa_{2}} A}{\left(27 A^{4} \varepsilon^{2}+24 A^{2} \alpha \varepsilon+\alpha^{2}\right)^{2}}
$$$$
\lambda_{1}=-\frac{27101207}{2} A^{8} \alpha^{4} \varepsilon^{4}+\frac{828547}{6} A^{4} \alpha^{6} \varepsilon^{2}-\frac{34744149}{2} A^{12} \alpha^{2} \varepsilon^{6}-\frac{4808853}{2} A^{6} \alpha^{5} \varepsilon^{3}
$$$$
-\frac{49237215}{2} A^{10} \alpha^{3} \varepsilon^{5}+\frac{473}{6} A^{2} \alpha^{7} \varepsilon-\frac{4093119}{2} A^{14} \alpha \varepsilon^{7}-\frac{3941}{6} A^{12} \Gamma^{2} \varepsilon^{6}
$$$$
+15724800 A^{14} \Gamma \varepsilon^{7}+\frac{47}{243} A^{2} \Gamma^{2} \alpha^{5} \varepsilon-\frac{2488}{3} A^{2} \Gamma \alpha^{6} \varepsilon+\frac{2}{3} \alpha^{8}+\frac{3448575}{2} A^{16} \varepsilon^{8}
$$$$
-\frac{2069474}{3} A^{4} \Gamma \alpha^{5} \varepsilon^{2}+\frac{935}{18} A^{4} \Gamma^{2} \alpha^{4} \varepsilon^{2}+6126560 A^{6} \Gamma \alpha^{4} \varepsilon^{3}-\frac{21596}{81} A^{6} \Gamma^{2} \alpha^{3} \varepsilon^{3}
$$$$
+36949526 A^{8} \Gamma \alpha^{3} \varepsilon^{4}-\frac{2659}{2} A^{8} \Gamma^{2} \alpha^{2} \varepsilon^{4}+68335560 A^{10} \Gamma \alpha^{2} \varepsilon^{5}-\frac{15017}{9} A^{10} \Gamma^{2} \alpha \varepsilon^{5}
$$$$
+53917182 A^{12} \Gamma \alpha \varepsilon^{6}-\frac{26}{3} \Gamma \alpha^{7}+\frac{1}{486} \Gamma^{2} \alpha^{6} .
$$

$$
\eta_{1}=\frac{\left(27 A^{4} \varepsilon^{2}+24 A^{2} \alpha \varepsilon+\alpha^{2}\right)^{2}}{\Gamma^{2} \sqrt{\lambda_{1}}}
$$




$$
\begin{gathered}
\lambda_{2}=53648 A^{8} \alpha^{4} \varepsilon^{4}+\frac{2697568}{3} A^{4} \alpha^{6} \varepsilon^{2}-24564672 A^{12} \alpha^{2} \varepsilon^{6}+3124128 A^{6} \alpha^{5} \varepsilon^{3}-14036448 A^{10} \alpha^{3} \varepsilon^{5} \\
-\frac{16096}{3} A^{2} \alpha^{7} \varepsilon-16936992 A^{14} \alpha \varepsilon^{7}-\frac{371}{3} A^{12} \Gamma^{2} \varepsilon^{6}+3241728 A^{14} \Gamma \varepsilon^{7}-\frac{806}{243} A^{2} \Gamma^{2} \alpha^{5} \varepsilon \\
+\frac{132640}{3} A^{2} \Gamma \alpha^{6} \varepsilon-\frac{80}{3} \alpha^{8}-4240512 A^{16} \varepsilon^{8}-\frac{9696352}{3} A^{4} \Gamma \alpha^{5} \varepsilon^{2}+\frac{16375}{81} A^{4} \Gamma^{2} \alpha^{4} \varepsilon^{2} \\
-14300672 A^{6} \Gamma \alpha^{4} \varepsilon^{3}+\frac{70108}{81} A^{6} \Gamma^{2} \alpha^{3} \varepsilon^{3}-20516512 A^{8} \Gamma \alpha^{3} \varepsilon^{4}+\frac{11015}{9} A^{8} \Gamma^{2} \alpha^{2} \varepsilon^{4} \\
-8157024 A^{10} \Gamma \alpha^{2} \varepsilon^{5}+\frac{6166}{9} A^{10} \Gamma^{2} \alpha \varepsilon^{5}+4567392 A^{12} \Gamma \alpha \varepsilon^{6}+\frac{800}{3} \Gamma \alpha^{7}-\frac{5}{243} \Gamma^{2} \alpha^{6} \\
\eta_{2}=\frac{\left(27 A^{4} \varepsilon^{2}+24 A^{2} \alpha \varepsilon+\alpha^{2}\right)^{2}}{\Gamma^{2} \sqrt{\lambda_{2}}}
\end{gathered}
$$

With regard to Eqs.(47-56), the constant coefficients of Eq.(37) can be rewritten as follows:

$$
\begin{aligned}
& \omega=\frac{1}{18} \sqrt{324} \sqrt{\Gamma}, b_{0}=\frac{1}{4} \zeta_{1}, b_{1}=\frac{1}{5} \zeta_{2} \\
& b_{2}=-\frac{1458}{5} \frac{A\left(3 A^{4} \varepsilon^{2}-5 A^{2} \Gamma \varepsilon+4 A^{2} \alpha \varepsilon+\frac{1}{2916} \Gamma^{2}-5 \alpha \Gamma+\alpha^{2}\right)}{\Gamma^{2}} \\
& \varphi_{0}=-\frac{1}{2}\left(-1+\operatorname{csgn}\left(\eta_{1}\left(3 A^{4} \varepsilon^{2}-13 A^{2} \Gamma \varepsilon+4 A^{2} \alpha \varepsilon+\frac{1}{324} \Gamma^{2}-13 \Gamma \alpha+\alpha^{2}\right)\right)\right) \pi \\
& \varphi_{1}=\frac{1}{2}\left(1+\operatorname{csgn}\left(\eta_{2}\left(3 A^{4} \varepsilon^{2}-10 A^{2} \Gamma \varepsilon+4 A^{2} \alpha \varepsilon+\frac{1}{1296} \Gamma^{2}-10 \Gamma \alpha+\alpha^{2}\right)\right)\right) \pi \\
& \varphi_{2}=\pi .
\end{aligned}
$$

By considering the following physical quantities for this equation:

$$
\alpha=3, A=1.5, \varepsilon=0.5 \text {. }
$$

After substituting the mentioned physical quantities into Eqs.(47-56) and Eq.(57), then by substituting the final obtained values in Eq. (37) the solution of the mentioned will be obtained as follows

$$
\begin{aligned}
x(t)= & 1.504328625 \cos (1.983553268 t)+0.02145246657 \cos (3.967106536 t+3.141592654) \\
& -0.01712384134 \cos (5.950659804 t+3.141592654) .
\end{aligned}
$$

\section{Conclusion}

The current study presents that AGM is efficient and accurate in comparison with numerical method (Ruge-Kutta, $R_{4}$ ) and by utilizing this method through mentioned examples it would be obvious that AGM have the strong ability to solve nonlinear equations especially vibrational equations.

In Example 1 of this paper investigation has been done through Van Der Pol oscillator which is under many studies in decades, solving the afore-mentioned equation by AGM with only one term of assumed function as the answer of equation (according to basic idea of AGM), results of this procedure shows the accuracy and simplicity of AGM which is obvious in the relevant plots and table, we could certainly claim that the answer that is obtained by this method is exactly the same as numerical solution.

In example 2 of this paper investigation has been done through another nonlinear equation with physical constant 
coefficients, for solving that equation our assumed function for the answer of the equation include three terms, which would announce the high precision of this method and the results from relevant plots and table certainly presents the accuracy of AGM that has just minor different in comparison with numerical method.

In AGM it is very convenient and easy to find the solution of the differential equation and also the angular frequency $(\omega)$ simultaneously only by selecting a function as the answer of the differential equation in regard to the kind of the operation of the system. In AGM, the angular frequency $(\omega)$ is always obtained with the other unknown parameters of the oscillating systems such as initial vibrational phase, etc. in contrast to HPM and VIM and other semi-analytical methods. Therefore, it is logical to say that AGM is a very applicable and suitable approach for solving nonlinear differential equations. In addition to the afore-mentioned explanations after applying initial conditions on the considered answer, we exit from the field of differential equation into a set of algebraic equations. Then, by solving a set of algebraic equations which is a simple procedure, the constant coefficients of the considered answer and also the angular frequency can easily be obtained.

Table 1: Nomenclature

\begin{tabular}{|l|l|}
\hline $\boldsymbol{u}(\boldsymbol{t})$ & Displacement(M1) \\
\hline $\boldsymbol{x}(\boldsymbol{t})$ & Displacement(M2) \\
\hline$\alpha$ & Constant parameter \\
\hline$\varepsilon$ & Constant parameter \\
\hline $\boldsymbol{M}_{1}$ & Mass 1 \\
\hline $\boldsymbol{M}_{2}$ & Mass 2 \\
\hline $\boldsymbol{A}$ & Vibration Amplitude \\
\hline $\boldsymbol{\omega}$ & Angular frequency \\
\hline $\boldsymbol{L}$ & Length \\
\hline $\boldsymbol{A G M}$ & Akbari-Ganji's Method \\
\hline $\boldsymbol{C}_{1}$ & Damper coefficient \\
\hline $\boldsymbol{C}_{2}$ & Damper coefficient \\
\hline
\end{tabular}

\section{References}

[1] M.R. Akbari, D.D.Ganji, A.Majidian, A.R. Ahmadi, Solving nonlinear differential equations of Vanderpol, Rayleigh and Duffing by AGM, Frontiers of Mechanical Engineering, Volume 9, Issue 2, pp. 177-190 (2015).

[2] S.Tahernejad Ledari, H.Mirgolbabaee, D.D.Ganji. Heat transfer analysis of a fin with temperature dependent thermal conductivity and heat transfer coefficient,New Trends in Mathematical Sciences,No.2,55-69 (2015).

[3] S.T.Ledari, H. Mirgolbabaee, D.D.Ganji. An assessment of a semi analytical AG method for solving two-dimension nonlinear viscous flow, International Journal of Nonlinear Analysis and Applications, 6(2): 47-64 (2014).

[4] S. Das, P. Gupta, Application of homotopy analysis method and homotopy perturbation method to fractional vibration equation, International Journal of Computer Mathematics, 88 (2), 430-441, (2011).

[5] T.Pirbodaghi, M.Ahmadian, M.Fesanghary, on the homotopy analysis method for non-linear vibration of beams, Mechanics Research Communications, 36 (2), 143-148, (2009).

[6] J. H. He, An improved amplitude-frequency formulation for nonlinear oscillators, International Journal of Nonlinear Sciences and Numerical Simulation, 9 (2), 211, (2008).

[7] S.S.Ganji, D.D.Ganji, H.Babazadeh, N.Sadoughi, Application of amplitude-frequency formulation to nonlinear oscillation system of the motion of a rigid rod rocking back, Mathematical Methods in the Applied Sciences, 33 (2), 157-166, (2010).

[8] A. Kimiaeifar, A. R. Saidia, A. R. Sohouli, D. D. Ganji, Analysis of modified Van der Polas oscillator using Heat's parameterexpanding methods, Curr. Appl. Phys., 10 (1), 279-283, (2010).

[9] M. G. Sfahani, A. Barari, M. Omidvar, S. S. Ganji, G. Domairry, Dynamic response of inextensible beams by improved energy balance method, Proceedings of the Institution of Mechanical Engineers, Part K Journal of multi-body Dynamics, 225 (1),66-73, (2011). 
[10] M. Momeni, N. Jamshidi, A. Barari, D. D. Ganji, Application of Heat's energy balance method to duffing harmonic oscillators, International Journal of Computer Mathematics, 88, 135-144, (2011).

[11] J.K. Zhou, Differential Transformation and its Applications for Electrical Circuits, Huazhong Univ. Press, Wuhan, China, (1986).

[12] D.D. Ganji, H. Mirgolbabaei, Me. Miansari, Mo. Miansari, Application of homotopy perturbation method to solve linear and non-linear systems of ordinary differential equations and differential equation of order three, Journal of Applied Sciences, 8(7), 1256-1261, (2008).

[13] H. Mirgolbabaei, D. D. Ganji, H. Taherian, Soliton solution of the Kadomtse-Petviashvili Equation by homotopy perturbation method, World Journal of Modeling and Simulation, 5(1), 38-44, (2009).

[14] S.Abbasbandy. A numerical solution of Blasius equation by Adomian's decomposition method and comparison with homotopy perturbation method. Chaos, Solitons and Fractals. 31: 257-260, (2007).

[15] Adomian, G. Solving Frontier Problems of Physics. The Decomposition Method. Kluwer Academic Publishers, Boston, MA. (1994).

[16] Ji-Huan He and Xu-Hong Wu. "Exp-function method for nonlinear wave equations"Chaos. Solitos \& Fractals. Volume 30, Issue 3, November2006, 700-708, (2006).

[17] Xu-Hong WU and Ji-Huan He. ”Solitary solutions, periodic solutions and compacton-like solutions using the Exp-function method "Computers \& mathematics with applicatios, Volume54, Issue 7-8,966- 986, (2007).

[18] SelÃßuk Kutluay and Alaattin Esen, Exp-function Method for Solving the General Improved KdV Equation. International Journal of Nonlinear Sciences and Numerical Simulation. Volume 10, Issue 6, 717-726, (2009).

[19] Jin-Rong Chang. The exp-function method and generalized solitary solutions. Computers \& Mathematics with Applications; 61:2081-2084. Pp.2081-2084, (2011).

[20] S. Ghafoori, M. Motevalli, M.G. Nejad, F. Shakeri, D.D. Ganji, M. Jalaal, Efficiency of differential transformation method for nonlinear oscillation: Comparison with HPM and VIM, January (2011)

[21] J.H. He, X.H. Wu, Construction of solitary solution and compacton-like solution by variational iteration method, Chaos, Solitons Fractals 29, 108-113, (2006).

[22] A. Barari, M. Omidvar, Abdoul R. Ghotbi, D.D. Ganji. Application of Homotopy Perturbation Method and Variational Iteration Method to Nonlinear Oscillator Differential Equations. DOI 10.1007/s10440-008-9248-9. Springer 2008.

[23] S.H. Behirya, H. Hashisha, I.L. El-Kallab, A. Elsaida. A new algorithm for the decomposition solution of nonlinear differential equations. Computers and Mathematics with Applications 54, 459-466, (2007).

[24] D.D. Ganji, A.R. Sahouli, M. Famouri. A new modification of Heat's homotopy perturbation method for rapid convergence of nonlinear undamped oscillators. DOI 10.1007/s12190-008-0165-x. Springer (2009).

\section{Appendix}

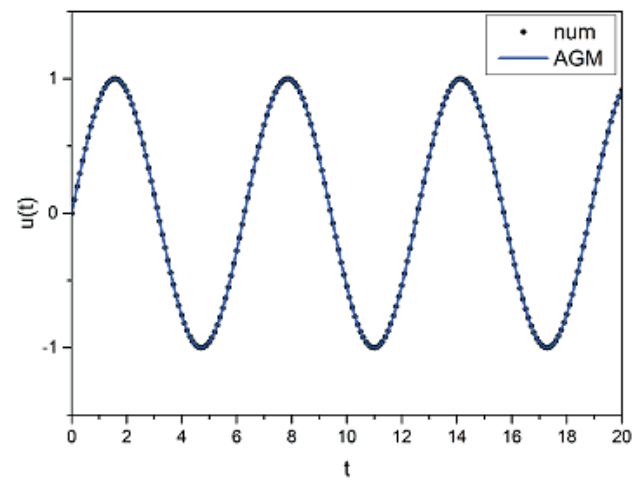

Fig. 3: A comparison between AGM and numerical method of example 1. 


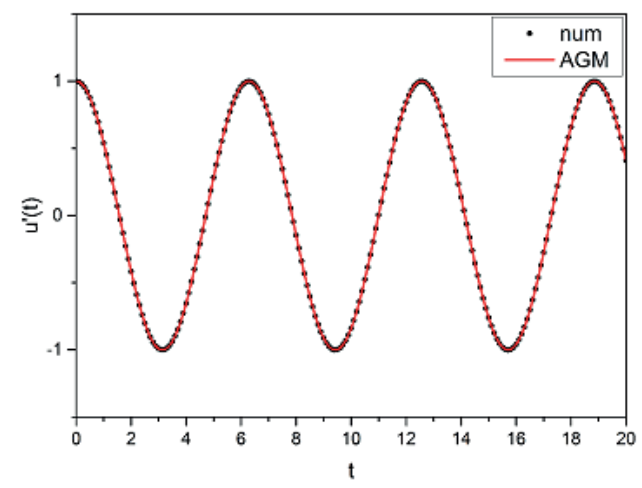

Fig. 4: A comparison between the first derivatives of AGM and numerical method of example 1.

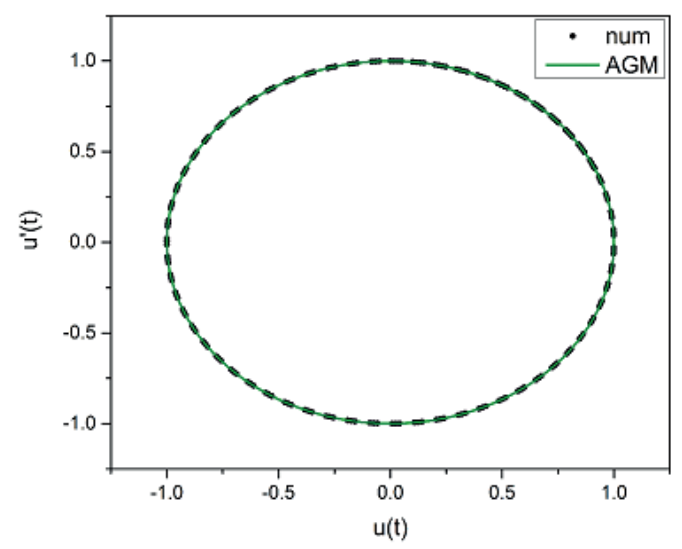

Fig. 5: A comparison between the obtained $u(t)$ and $u^{\prime}(t)$ by AGM and numerical method of example 1 .

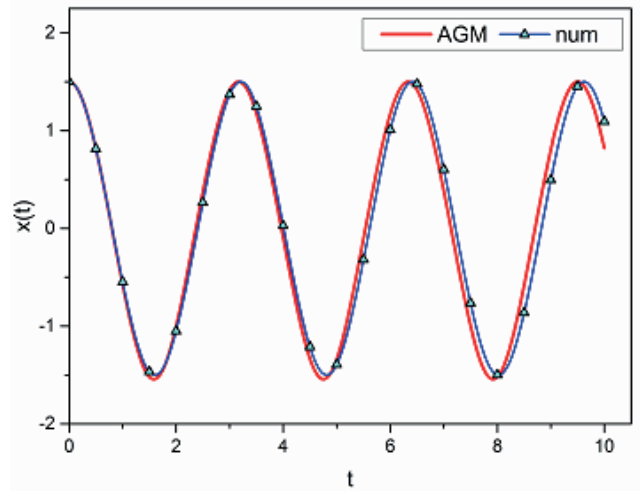

Fig. 6: A comparison between AGM and numerical method of example 2. 


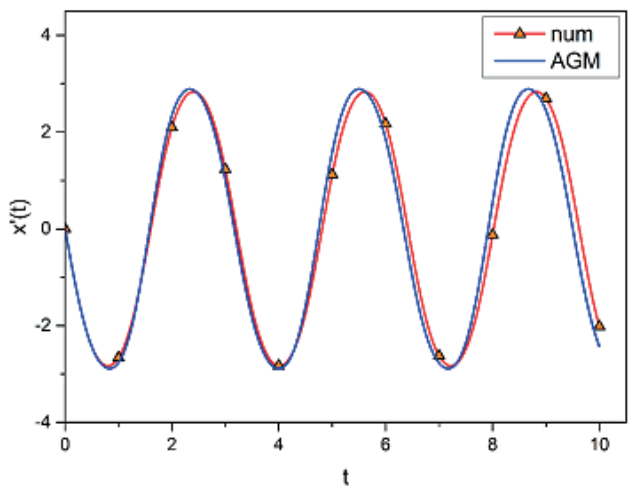

Fig. 7: A comparison between the first derivatives of AGM and numerical method of example 2.

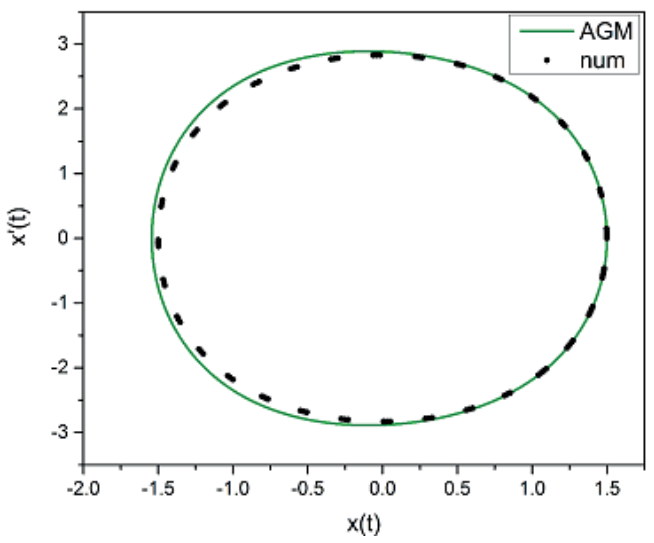

Fig. 8: A comparison between the obtained $x(t)$ and $x^{\prime}(t)$ by AGM and numerical method of example 2.

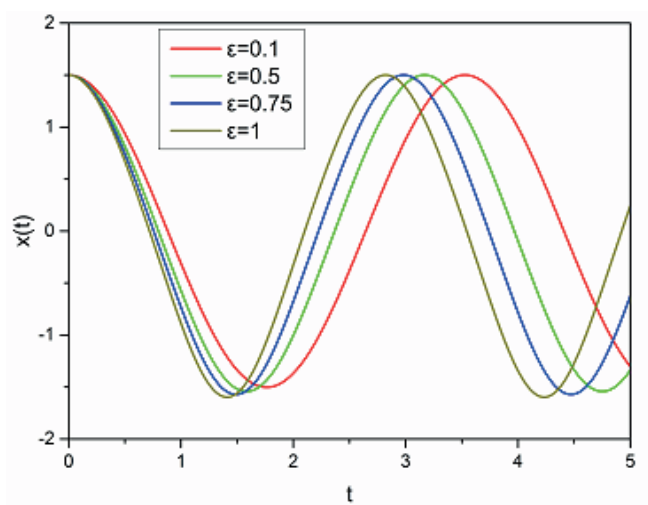

Fig. 9: A comparison amongst the obtained $x(t)$ by AGM for various amounts of $\varepsilon$ by assumption of $\alpha=3, A=1.5$ in example 2 . 


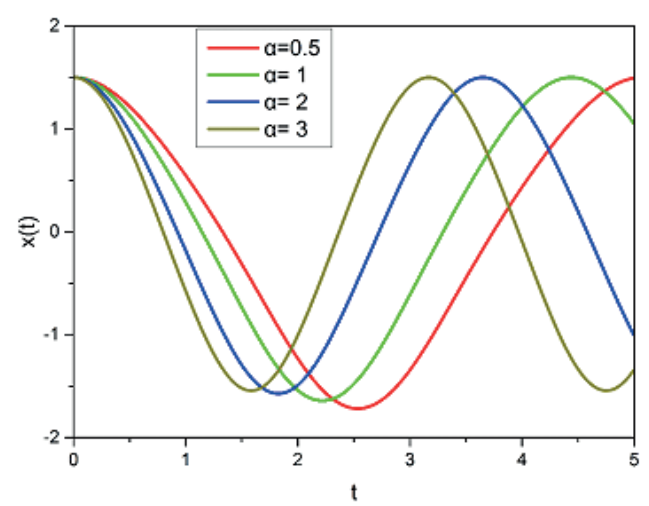

Fig. 10: A comparison amongst the obtained $x(t)$ by AGM for various amounts of $\alpha$ by assumption of $\varepsilon=0.5, A=1.5$ in example 2.

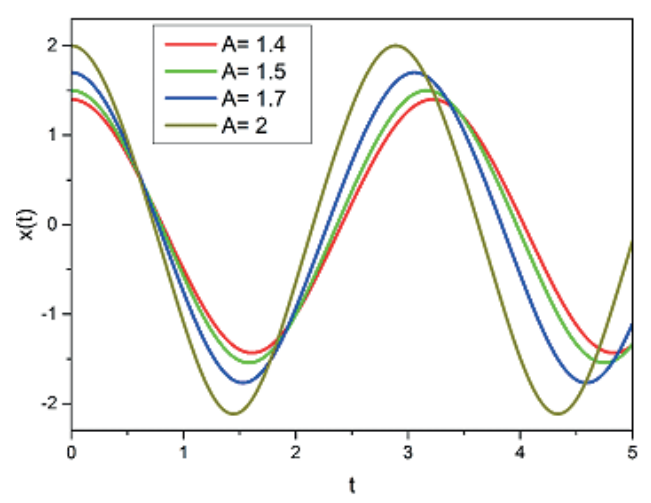

Fig. 11: A comparison amongst the obtained $x(t)$ by AGM for various amounts of $A$ by assumption of $\alpha=3, \varepsilon=0.5$ in example 2 .

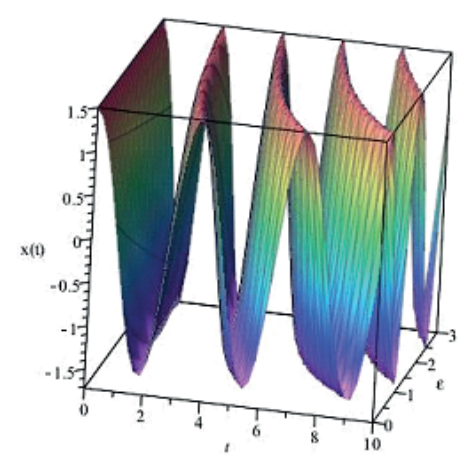

Fig. 12: A comparison amongst the obtained $x(t)$ by AGM for various ranges of $\varepsilon$ by assumption of $\alpha=3, A=1.5$ in example 2. 


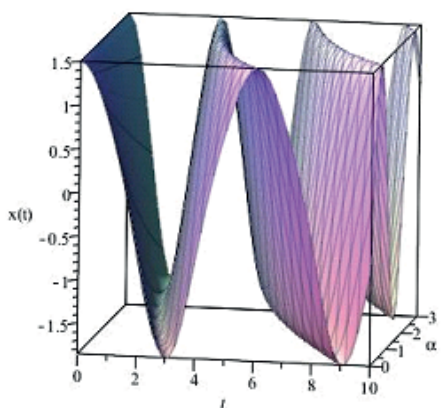

Fig. 13: A comparison amongst the obtained $x(t)$ by AGM for various ranges of $\alpha$ by assumption of $\varepsilon=0.5, A=1.5$ in example 2.

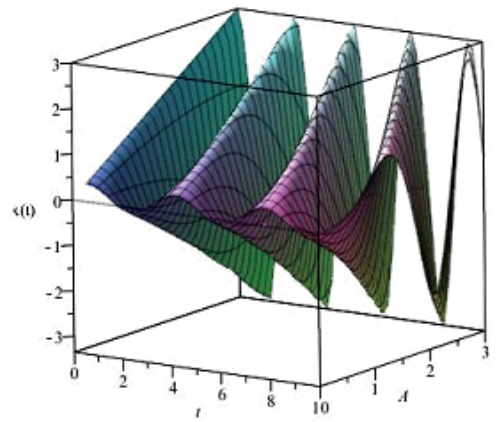

Fig. 14: A comparison amongst the obtained $x(t)$ by AGM for various ranges of $A$ by assumption of $\alpha=3, \varepsilon=0.5$ in example 2.

\begin{tabular}{|l|l|l|l|l|l|l|}
\hline t & $\mathbf{0}$ & $\mathbf{4}$ & $\mathbf{8}$ & $\mathbf{1 2}$ & $\mathbf{1 6}$ & $\mathbf{2 0}$ \\
\hline num & 0 & -0.75680 & 0.98936 & -0.53657 & -0.28790 & 0.91295 \\
\hline AGM & 0 & -0.75680 & 0.98935 & -0.53657 & -0.28790 & 0.91294 \\
\hline error & 0 & 0 & 0.00001 & 0 & 0 & 0.00001 \\
\hline
\end{tabular}

Table 2: The obtained results in accordance with the Numerical Solution and AGM according to Example 1.

\begin{tabular}{|l|l|l|l|l|l|l|}
\hline t & $\mathbf{0}$ & $\mathbf{2}$ & $\mathbf{4}$ & $\mathbf{6}$ & $\mathbf{8}$ & $\mathbf{1 0}$ \\
\hline num & 1.5 & -1.05162 & 0.02844 & 1.00878 & -1.49875 & 1.09296 \\
\hline AGM & 1.5 & -1.00501 & -0.09531 & 1.17157 & -1.52054 & 0.82200 \\
\hline error & 0 & -0.04661 & 0.12375 & -0.16279 & 0.02179 & 0.27096 \\
\hline
\end{tabular}

Table 3: The obtained results in accordance with the Numerical Solution and AGM according to Example 2. 